\title{
THERMODYNAMIC ANALYSIS OF AN HYDROMAGNETIC INTERNAL HEAT GENERATING VARIABLE-VISCOSITY CHANNEL FLOW WITH NON-UNIFORM WALL TEMPERATURE
}

\author{
Anthony R. Hassan 1, 2, * \\ ${ }^{1}$ Department of Mathematical Sciences, University of South Africa, \\ Pretoria 0003, South Africa \\ ${ }^{2}$ Department of Mathematics, Tai Solarin University of Education, \\ Ijagun, Ogun State, Nigeria \\ *Corresponding author; E-mail: anthonyhassan72@yahoo.co.uk
}

(Received July 31, 2017; Accepted October 17, 2017)

\begin{abstract}
This study examines the influence of magnetic intensity and internal heat generation on a flow system with variable-viscosity channel with varying wall temperatures. The fluid is assumed to be steady, incompressible with both the variable viscosity and internal heat generation to be a linear function of temperature. The approximate solutions are secured by using Adomian decomposition method (ADM) for the momentum and energy distributions as well as the rate of entropy generation and other thermophysical aspects of the flow regime under the impact of both the magnetic influence and internal heat generation are illustrated in tables and graphs.
\end{abstract}

Keywords: Hydromagnetic; internal heat generation; variable-viscosity; non-uniform wall temperature; entropy generation and Adomian decomposition method (ADM).

\section{INTRODUCTION}

Various researches on hydromagnetic fluid flow have been of high interest to scientists and engineers for the numerous applications and relevance in geophysics, engineering, industry and technology. For instance, PRASAD and KUMAR (2011) considered hydromagnetic flow within porous medium between two permeable beds in the presence of exponenttially decaying pressure gradient because of its widespread industrial and environmental applications. To support the study, the relevance in geothermal, oil reservoir engineering and astrophysics, to mention a few have been highlighted in BAOKU et al. (2012), where the paired effects of thermal radiation and magnetic field on the hydromagnetic Couette flow within porous medium has been investigated. Recently, HASSAN and MARITZ (2017b) scrutinized the effects of heat source on hydromagnetic Poiseuille fluid flow between two parallel plates stating the applications in many industrial and engineering processes especially in the purification of crude oil magneto-hydrodynamics generators. Other studies on hydromagnetic fluids can be found extensively in SARAVANAN and KANDASWAMY (2004), SAHA and ChaKRABARTI (2013), ZElalem et al. (2013), Kim (2014), XiAO and KiM 
(2014), HASSAN and GBADEyAn (2015a,b), KRishnAmurthy et al. (2015), MAKINDE et al. (2015), HASSAN and MARITZ (2016a,b) and UWANTA and HAMZA (2016).

Moreover, extensive survey with experimental, theoretical and numerical approaches on fluid behaviour with so many thermo physical properties which varies from one to many properties such as in MAKINDE et al. (2015) that employed laws of thermodynamics to examine the behaviour of fluid flow on thermal decomposition in hydromagnetic variable viscosity Couette flow in a rotating system with hall current. On the other hand, SHEIKHOLESLAMI et al. (2016) investigated heat and mass transfer characteristic involving unsteady Nano fluid flow between parallel plates. However, flow within parallel plates with different characteristics such as through porous medium in HASSAN and MARITZ (2016b) together with magnetic and thermal radiation effect as in Baoku et al. (2012). Also, variable - viscosity as found in MAKINDE (2008) and HASSAN and MARITZ (2017a) with non-uniform wall temperature. Other properties include heat source as found in ELBASHBESHY and BAZID (2004), El-Amin (2004), JHA and AJiBADE (2009), OlANREWAJU et al. (2011), HASSAN and GBADEYAN (2015b) and HASSAN and MARITZ (2016a).

Furthermore, the combination of two or more effects on fluid flow need serious attention, especially when heat source is involved because heat is normally produced when fluid particles interact and may alter the temperature distribution as well as the particle deposition rate as discussed in HASSAN and MARITZ (2017b). Motivated by this study, brought the idea to extend the study in MAKINDE (2008), by incorporating the impacts of heat source and magnetic strength on the irreversibility flow of a variable - viscosity fluid through a channel with non-uniform wall temperature as well as on the entropy generation rate and other thermophysical properties of the flow regime. This study is significant to technological applications such as in biomedical engineering, material and food processing. The coupled differential equations governing the fluid motion and energy are secured by using semi-analytical decomposition method named after G. Adomian. This particular method has been proved to be efficient for obtaining solutions to differential equations and converge rapidly with sizeable number of iterations. Further studies on ADM are extensively discussed in WAZWAZ (1999, 2000), WAZWAZ and EL-SAYED (2001) and RAY (2014).

\section{MATHEMATICAL MODEL}

Consider the steady flow of an hydromagnetic, incompressible and electrically conducting fluid flowing in the $\bar{x}$ - direction through fixed plates of width $(a)$ and length $(L)$, with non-uniform wall temperatures at the upper $\left(T_{u}\right)$ and lower $\left(T_{L}\right)$ plates under the influence of a constant pressure gradient together with that of a transverse magnetic field strength $\left(B_{0}\right)$ as displayed in figure 1 .

Figure 1: Geometry of the Problem

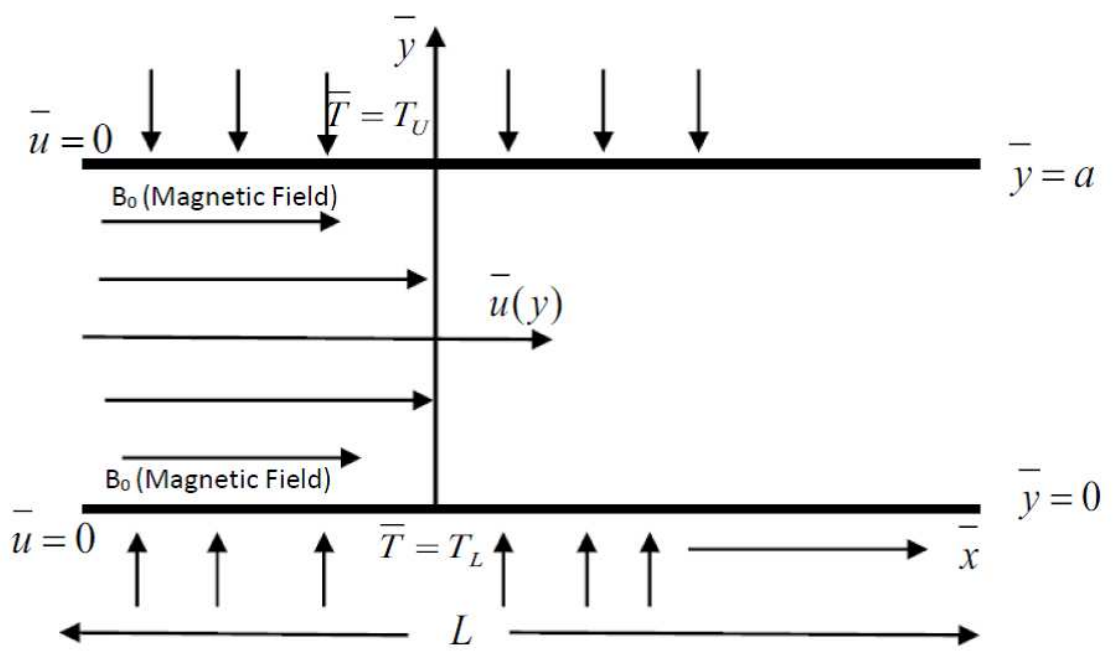


The temperature dependent viscosity $(\bar{\mu})$ as described in MAKINDE (2008), HASSAN and MARITZ (2017b), and the heat source $(\bar{\delta})$ that is assumed to be a linear function of temperature as in JHA and AJIBADE (2009), HASSAN and GBADEYAN (2015b) and HASSAN and MARITZ (2016a) can be expressed as

$$
\bar{\mu}=\mu_{0}\left[1-\beta\left(\bar{T}-T_{L}\right)\right] \text { and } \bar{\delta}=\delta_{0}\left[\bar{T}-T_{L}\right]
$$

where $\mu_{0}$ is the fluid dynamic viscosity at the lower wall temperature $\left(T_{L}\right), \beta$ is the viscosity - variation parameter, $\delta_{0}$ represents the dimensional heat generation coefficient and $\bar{T}$ is the fluid temperature.

Neglecting the reactant consumption of the fluid; the continuity, momentum (along $x$ and $y$ axes) and energy equations governing the fluid flow in non-dimensionless form may be given as in MAKINDE (2008), HASSAN and GBADEYAN (2015b) and HASSAN and MARITZ (2017b):

$$
\begin{aligned}
& \frac{\partial \bar{u}}{\partial \bar{x}}+\frac{\partial \bar{v}}{\partial \bar{y}}=0 \\
& \rho\left[\bar{u} \frac{\partial \bar{u}}{\partial \bar{x}}+\bar{v} \frac{\partial \bar{u}}{\partial \bar{y}}\right]=-\frac{\partial \bar{p}}{\partial \bar{x}}+2 \frac{\partial}{\partial \bar{x}}\left(\bar{\mu} \frac{\partial \bar{u}}{\partial \bar{x}}\right)+\frac{\partial}{\partial \bar{y}}\left[\bar{\mu}\left(\frac{\partial \bar{u}}{\partial \bar{y}}+\frac{\partial \bar{v}}{\partial \bar{x}}\right)\right]-\sigma B_{0}^{2} \bar{u} \\
& \rho\left[\bar{u} \frac{\partial \bar{v}}{\partial \bar{x}}+\bar{v} \frac{\partial \bar{v}}{\partial \bar{y}}\right]=-\frac{\partial \bar{p}}{\partial \bar{y}}+2 \frac{\partial}{\partial \bar{y}}\left(\bar{\mu} \frac{\partial \bar{v}}{\partial \bar{y}}\right)+\frac{\partial}{\partial \bar{x}}\left[\bar{\mu}\left(\frac{\partial \bar{u}}{\partial \bar{y}}+\frac{\partial \bar{v}}{\partial \bar{x}}\right)\right] \\
& \rho c_{p}\left[\bar{u} \frac{\partial \bar{T}}{\partial \bar{x}}+\bar{v} \frac{\partial \bar{T}}{\partial \bar{y}}\right]=k\left[\frac{\partial^{2} \bar{T}}{\partial \bar{x}}+\bar{v} \frac{\partial^{2} \bar{T}}{\partial \bar{y}^{2}}\right] \\
& +\bar{\mu}\left[2\left(\frac{\partial \bar{u}}{\partial \bar{x}}\right)^{2}+2\left(\frac{\partial \bar{v}}{\partial \bar{y}}\right)^{2}+\left(\frac{\partial \bar{u}}{\partial \bar{y}}+\frac{\partial_{\bar{v}}}{\partial \bar{x}}\right)^{2}\right]+\sigma B_{0}^{2} \bar{u}^{2}+\delta_{0}\left(\bar{T}-T_{L}\right)
\end{aligned}
$$

where $\rho$ stands for the fluid density, $k$ for thermal conductivity, $p$ is the pressure, $\sigma$ for electrical conductivity, $c_{p}$ is the specific heat at constant pressure, $u$ represent the axial velocity, $v$ is the normal velocity, also, $x$ and $y$ are distances measured in the streamline and normal direction respectively. It is worthy to note that the bar on each variable represents the non-dimensionless form. However, the additional terms in equations (3) and (5) represent the magnetic intensity influence and internal heat source on the flow regime as in ZELALEM et al. (2013), HASSAN and GBADEYAN (2015a,b) and HASSAN and MARITZ (2016a).

However, the rate of entropy which is an account of disorderliness in a system is also a way of providing another variable that can be used to describe the state of flow regime. Following WoOdS (1975), MAKINDE (2008), HASSAN and GBADEYAN (2015a,b) and HASSAN and MARITZ (2016a, 2017b), the general equation for the rate of entropy generation $\left(S^{m}\right)$ under the influence of magnetic field is expressed as:

$$
\mathrm{S}^{\mathrm{m}}=\frac{k}{T_{L}}\left(\frac{\partial \bar{T}}{\partial \bar{y}}\right)^{2}+\frac{\bar{\mu}}{T_{L}}\left(\frac{\partial \bar{u}}{\partial \bar{y}}\right)^{2}+\frac{\sigma B_{0}^{2} \bar{u}}{T_{L}}
$$

Also, the first term in (6) represents the irreversibility due to heat transfer, the second term is the entropy generation due to viscous dissipation and the third term is the local entropy generation due to the effects of magnetic strength. 
Introducing the following non - dimensional quantities in equations (1) - (6) as follows:

$$
\begin{aligned}
& y=\frac{\bar{y}}{\varepsilon L}, x=\frac{\bar{x}}{L}, u=\frac{\bar{u}}{U}, v=\frac{\bar{v}}{\varepsilon U}, \varepsilon=\frac{a}{L}, \mu=\frac{\bar{\mu}}{\mu_{0}}, G=-\frac{d P}{d x}, P e=\frac{\rho c_{p} L U}{k}, \\
& P e=\frac{\rho L U}{\mu_{0}}, T=\frac{\bar{T}-T_{L}}{T_{u}-T_{L}}, p=\frac{\varepsilon^{2} L \bar{p}}{\mu_{0} U}, \alpha=\beta\left(T_{u}-T_{L}\right), B r=\frac{\mu_{0} U^{2}}{k\left(T_{u}-T_{L}\right)}, \\
& H^{2}=\frac{\sigma B_{0}{ }^{2} a^{2}}{\mu_{0}} \text { and } \delta=\frac{\delta_{0} a^{2}}{k}
\end{aligned}
$$

where $G$ is the pressure gradient, $T_{u}$ is the upper wall temperature, $\varepsilon$ is the is the channel aspect ratio and $U$ is the velocity scale. In addition to that, $\alpha$ and $\delta$ respectively stand for the parameters of viscosity - variation and internal heat generation; while $\mathrm{Br}, \mathrm{Re}, \mathrm{Pe}$ and $\mathrm{H}$ are numbers respectively standing for Brinkman, Reynolds, Peclet and Hartmann. fluid flow:

Introducing (7) into equations (1) - (6), the following are the equations regulating the

$$
\begin{gathered}
\frac{\partial u}{\partial x}+\frac{\partial v}{\partial y}=0 \\
\varepsilon^{2} \operatorname{Re}\left[\frac{\partial u}{\partial x}+v \frac{\partial u}{\partial y}\right]=G+2 \varepsilon^{2}\left(\mu \frac{\partial u}{\partial x}\right)+\frac{\partial}{\partial y}\left[\mu\left(\frac{\partial u}{\partial y}+\varepsilon^{2} \frac{\partial u}{\partial x}\right)\right]-H^{2} u \\
\varepsilon^{4} \operatorname{Re}\left[u \frac{\partial v}{\partial x}+v \frac{\partial v}{\partial y}\right]=-\frac{\partial p}{\partial y}+2 \varepsilon^{2}\left(\mu \frac{\partial v}{\partial y}\right)+\varepsilon^{2} \frac{\partial}{\partial x}\left[\mu\left(\frac{\partial u}{\partial y}+\varepsilon^{2} \frac{\partial v}{\partial x}\right)\right] \\
\varepsilon^{2} P e\left[u \frac{\partial T}{\partial x}+v \frac{\partial T}{\partial y}\right]=\varepsilon^{2} \frac{\partial^{2} T}{\partial x^{2}}+\frac{\partial^{2} T}{\partial y^{2}}+B r\left[\mu \varphi+H^{2} u^{2}\right]+\delta T \\
\text { where } \varphi=2 \varepsilon^{2}\left(\frac{\partial u}{\partial x}\right)^{2}+2 \varepsilon^{2}\left(\frac{\partial v}{\partial y}\right)^{2}+\left(\frac{\partial u}{\partial y}+\varepsilon^{2} \frac{\partial v}{\partial x}\right)^{2}
\end{gathered}
$$

Since the channel aspect ratio is narrow, that is, $0<\varepsilon<<1$, the lubrication estimation essentially on asymptotic simplification of the governing equations (8) - (11) is invoked following MAKINDE (2008) and HASSAN and MARITZ (2017b) to obtain:

$$
\begin{aligned}
& \frac{\partial}{\partial y}\left(\mu \frac{\partial u}{\partial y}\right)-H^{2} u+G+O\left(\varepsilon^{2}\right)=0 \\
& -\frac{\partial p}{\partial y}++O\left(\varepsilon^{2}\right)=0 \\
& \frac{\partial^{2} T}{\partial y^{2}}+B r\left[\mu+\left(\frac{\partial u}{\partial y}\right)^{2}+H^{2} u^{2}\right]+\delta T+O\left(\varepsilon^{2}\right)=0
\end{aligned}
$$

where $\mu=1-\alpha T$ with the following boundary conditions at the upper wall of the channel as:

$$
u=0, T=1 \quad \text { at } \quad y=1
$$

and at the lower wall of the channel as:

$$
u=0, T=0 \quad \text { at } \quad y=0
$$

Note that (15) and (16) indicate that the temperature at both fixed upper and lower walls are not the same. 
Finally, the dimensionless form of (6) using the existing variables in (7) is expressed as follows:

$$
\mathrm{N}_{\mathrm{s}}=\frac{\mathrm{S}^{\mathrm{m}} a^{2} T_{L}^{2}}{k\left(T_{u}-T_{L}\right)^{2}}=\left(\frac{\partial T}{\partial y}\right)^{2}+\frac{B r}{\Omega}\left[\left(\mu \frac{\partial u}{\partial y}\right)^{2}+H^{2} u^{2}\right]
$$

where $\Omega$ stands for the wall temperature difference parameter.

\section{METHOD OF SOLUTION [Adomian decomposition method (ADM)]}

The ADM is a semi-analytical method of solving differential equations which has been proved to be efficient and converge with sizeable number of iterations. The literature is rich in the following WAZWAZ (2000), WAZWAZ and EL-SAYED (2001), HASSAN and GBADEYAN (2015a,b) and HASSAN and MARITZ (2016a).

Equations (12) and (14) are coupled equations that need to be solved simultaneously. It is good to note that (13), upon integration gives a constant pressure along the $y$-axis direction. Therefore, substituting $\mu$ which is equal to $1-\alpha T$ into the equations (12) and (14) subjected to the boundary conditions (15) and (16) and integrating appropriately to obtain the following couple equations:

$$
\begin{array}{r}
u(y)=a_{0} y-\frac{G}{2} y^{2}+\alpha \int_{0}^{y} T(y) \frac{\partial u}{\partial y} d Y+H^{2} \int_{0}^{y} \int_{0}^{y} u(y) d Y d Y \\
T(y)=b_{0} y-B r \int_{0}^{y} \int_{0}^{y}\left(\frac{\partial u}{\partial y}\right)^{2} d Y d Y+B r \alpha \int_{0}^{y} \int_{0}^{y} T(y)\left(\frac{\partial u}{\partial y}\right)^{2} d Y d Y \\
-B r H^{2} \int_{0}^{y} \int_{0}^{y}[u(y)]^{2} d Y d Y-\delta \int_{0}^{y} \int_{0}^{y} T(y) d Y d Y
\end{array}
$$

where $a_{0}=u^{\prime}(0)$ and $b_{0}=T^{\prime}(0)$ are constants of integration to be determined by using the boundary condition (15). However, to solve the coupled equations (18) and (19), we assume infinite series solutions in the form of:

$$
u(y)=\sum_{n=0}^{\infty} u_{n}(y) \quad \text { and } \quad T(y)=\sum_{n=0}^{\infty} T_{n}(y)
$$

such that when (20) is substituted in (18) and (19), we obtain,

$$
\begin{gathered}
u(y)=a_{0} y-\frac{G}{2} y^{2}+\alpha \int_{0}^{y}\left(\sum_{n=0}^{\infty} T_{n}(y)\right) \frac{\partial}{\partial y}\left(\sum_{n=0}^{\infty} u_{n}(y)\right) d Y+H^{2} \int_{0}^{y} \int_{0}^{y}\left(\sum_{n=0}^{\infty} u_{n}(y)\right) d Y d Y \\
T(y)=b_{0} y-B r \int_{0}^{y} \int_{0}^{y}\left(\frac{\partial}{\partial y}\left(\sum_{n=0}^{\infty} u_{n}(y)\right)\right)^{2} d Y d Y+B r \alpha \int_{0}^{y} \int_{0}^{y}\left(\sum_{n=0}^{\infty} T_{n}(y)\right)\left(\frac{\partial}{\partial y}\left(\sum_{n=0}^{\infty} u_{n}(y)\right)\right)^{2} d Y d Y \\
-B r H^{2} \int_{0}^{y} \int_{0}^{y}\left(\sum_{n=0}^{\infty} u_{n}(y)\right)^{2} d Y d Y-\delta \int_{0}^{y} \int_{0}^{y}\left(\sum_{n=0}^{\infty} T_{n}(y)\right) d Y d Y
\end{gathered}
$$

Here, in order to use ADM, we let non - linear terms in (21) and (22) be represented as:

$$
\begin{aligned}
& \sum_{n=0}^{\infty} A_{n}(y)=\left(\sum_{n=0}^{\infty} T_{n}(y)\right) \frac{\partial}{\partial y}\left(\sum_{n=0}^{\infty} u_{n}(y)\right), \sum_{n=0}^{\infty} B_{n}(y)=\left(\frac{\partial}{\partial y}\left(\sum_{n=0}^{\infty} u_{n}(y)\right)\right)^{2} \\
& \sum_{n=0}^{\infty} C_{n}(y)=\left(\sum_{n=0}^{\infty} T_{n}(y)\right)\left(\frac{\partial}{\partial y}\left(\sum_{n=0}^{\infty} u_{n}(y)\right)\right)^{2} \text { and } \sum_{n=0}^{\infty} D_{n}(y)=\left(\sum_{n=0}^{\infty} u_{n}(y)\right)^{2}
\end{aligned}
$$


where the respective components $A_{0}, A_{1}, A_{2}, \ldots, B_{0}, B_{1}, B_{2}, \ldots, C_{0}, C_{1}, C_{2}, \ldots$ and $D_{0}, D_{1}, D_{2}, \ldots$ are called Adomian polynomials. Then (23) is thereby expanded such that $A_{0}=T_{0}(y) u_{0}^{\prime}(y), A_{1}=T_{1}(y) u_{0}^{\prime}(y)+T_{0}(y) u_{1}^{\prime}(y), A_{2}=T_{2}(y) u_{0}^{\prime}(y)+T_{1}(y) u_{1}^{\prime}(y)+T_{0}(y) u_{2}^{\prime}(y)$, $B_{0}=u_{0}^{\prime}(y)^{2}, B_{1}=2 u_{0}^{\prime}(y) u_{1}^{\prime}(y), B_{2}=u_{1}^{\prime}(y)^{2}+2 u_{0}^{\prime}(y) u_{2}^{\prime}(y), \ldots$ $C_{0}=T_{0}(y) u_{0}^{\prime}(y)^{2}, C_{1}=u_{0}^{\prime}(y)\left(T_{1}(y) u_{0}^{\prime}(y)+2 T_{0}(y) u_{1}^{\prime}(y)\right)$, $C_{2}=T_{2}(y) u_{0}^{\prime}(y)^{2}+2 T_{1}(y) u_{1}^{\prime}(y) u_{0}^{\prime}(y)+T_{0}(y)\left(u_{1}^{\prime}(y)^{2}+2 u_{0}^{\prime}(y) u_{2}^{\prime}(y)\right), \ldots$ $D_{0}=u_{0}(y)^{2}, D_{1}=2 u_{0}(y) u_{1}(y), D_{2}=u_{1}(y)^{2}+2 u_{0}(y) u_{2}(y), \ldots$

With (23), the energy and motion equations respectively reduce to:

$$
\begin{aligned}
u(y)= & a_{0} y-\frac{G}{2} y^{2}+\alpha \int_{0}^{y} A_{n}(y) d Y+H^{2} \int_{0}^{y} \int_{0}^{y} u_{n}(y) d Y d Y \\
T(y)= & b_{0} y-B r \int_{0}^{y} \int_{0}^{y} B_{n}(y) d Y d Y+B r \alpha \int_{0}^{y} \int_{0}^{y} C_{n}(y) d Y d Y \\
& -B r H^{2} \int_{0}^{y} \int_{0}^{y} D_{n}(y) d Y d Y-\delta \int_{0}^{y} \int_{0}^{y} T_{n}(y) d Y d Y
\end{aligned}
$$

Then, following recursive relation with the zeroth component as already discussed in WAZWAZ (1999), WAZWAZ and EL-SAYED (2001), BABOLIAN and BIAZAR (2002) and RAY (2014), we obtain the following:

$$
\begin{aligned}
& u_{0}(y)=a_{0} y-\frac{G}{2} y^{2}, \quad T_{0}(y)=b_{0} y \\
& u_{n+1}(y)=\alpha \int_{0}^{y} A_{n}(y) d Y+H^{2} \int_{0}^{y} \int_{0}^{y} u_{n}(y) d Y d Y \\
& T_{n+1}(y)=-B r \int_{0}^{y} \int_{0}^{y} B_{n}(y) d Y d Y+B r \alpha \int_{0}^{y} \int_{0}^{y} C_{n}(y) d Y d Y \\
& -B r H^{2} \int_{0}^{y} \int_{0}^{y} D_{n}(y) d Y d Y-\delta \int_{0}^{y} \int_{0}^{y} T_{n}(y) d Y d Y, \quad n \geq 0
\end{aligned}
$$

Moreover, equations (27) - (28) are thereby coded on Mathematica software package, then substituting the results back into (20) to obtain the solutions for the velocity and temperature profiles which are hereby discussed in the next section because of the large volume of outputs.

Finally, for easy computations of equation (17), we assigned the first term, $\left(N_{1}\right)$ and other terms as $\left(N_{2}\right)$ such that:

$$
\mathrm{N}_{1}=\left(\frac{\partial T}{\partial y}\right)^{2} \text { and } \mathrm{N}_{2}=\frac{B r}{\Omega}\left[\left(\mu \frac{\partial u}{\partial y}\right)^{2}+H^{2} u^{2}\right]
$$

where $N_{1}$ is the irreversibility due to heat transfer and $N_{2}$ is the entropy generation rate due to the compound impacts of viscous dissipation and magnetic strength. However, it is essential to understand the supremacy of heat transfer irreversibility over fluid friction, as a result of that, BEJAN (1996) defined irreversibility distribution ratio as

$$
\phi=\frac{N_{2}}{N_{1}}
$$


Relation (30) shows that heat transfer has dominion when $0<\phi<1$ and fluid friction has dominion when $\phi>1$. But, when the rate of entropy production of heat transfer is equal to that of fluid friction, it implies that, $\phi=1$. As an alternative to irreversibility parameter, the Bejan number $(\mathrm{Be})$ which shows the contribution of both the heat transfer and fluid friction to entropy generation rate is defined as

$$
B e=\frac{N_{1}}{N_{s}}=\frac{1}{1+\phi} \quad \text { where } \quad 0 \leq B e \leq 1
$$

The expressions for (29) to (31) can be determined from the solutions in (20) using code on Mathematica software package as well.

\section{DISCUSSION OF RESULTS}

This section discussed the effects of the internal heat generation and magnetic intensity together with other important flow parameters on a variable-viscosity fluid flow with non-uniform wall temperature. Table 1 shows the rapid convergence of the series solutions for the numerical constants $a_{0}$ and $b_{0}$ from the equations (18) and (19) which converge at fifth iteration.

Table 1: Rapid convergence of the series solution for numerical constants $a_{0}$ and $b_{0}$.

\begin{tabular}{ccc}
\hline \multicolumn{3}{c}{$\alpha=0.1, H=0.5, G=1$ and $B r=10$} \\
\hline $\boldsymbol{n}$ & $a_{0}$ & $b_{0}$ \\
\hline $\mathbf{0}$ & 0.50000 & 1.00000 \\
$\mathbf{1}$ & 0.50143 & 1.44162 \\
$\mathbf{2}$ & 0.49860 & 1.45440 \\
$\mathbf{3}$ & 0.497877 & 1.45045 \\
$\mathbf{4}$ & 0.497882 & 1.44956 \\
$\mathbf{5}$ & 0.49792 & 1.4496 \\
$\mathbf{6}$ & 0.49792 & 1.4496 \\
\hline
\end{tabular}

Table 2 shows the comparison of numerical results of velocity and temperature profiles with previously obtained results in MAKINDE (2008), where perturbation method was used and the present result of which ADM was used. From the table, it is observed that the absolute error obtained had an average order of $10^{-4}$ which shows that ADM is also another efficient means of obtaining solutions to differential equations.

The velocity distributions of the fluid system are displayed in figures 2 to 5 for variations in $\alpha, \delta, B r$ and $H$ respectively. The graphs show that the maximum speed is obtained at the centerline of the flow channel of which the rising values of $\alpha, \delta$ and $\mathrm{Br}$ amount to greatest motion in the flow regime as in figures 2 to 4 while the reverse is noticed in figure 5 where the least speed is recorded with rising value of $H$. Of course, the rising values of $\alpha$, $\delta$ and $\mathrm{Br}$ allows the fluid to interact and hence increase the fluid internal energy by moving faster while the magnetic strength parameter $H$ has a retarding effect due to the presence of Lorentz forces across the flow channel. 
Table 2: Comparison of numerical results of velocity and temperature profiles.

$$
\delta=H=0 . \alpha=0.1, G=1, B r=10
$$

\begin{tabular}{ccccccc}
\hline $\boldsymbol{y}$ & $\boldsymbol{u}(\boldsymbol{y}) \mathbf{P M}$ & $\boldsymbol{u}(\boldsymbol{y})$ ADM & Absolute Error & $\boldsymbol{T}(\boldsymbol{y}) \mathbf{P M}$ & $\boldsymbol{T}(\boldsymbol{y})$ ADM & Absolute Error \\
\hline $\mathbf{0 . 0}$ & 0.000000 & 0.000000 & 0 & 0 & 0 & 0 \\
$\mathbf{0 . 1}$ & 0.046124 & 0.046186 & $6.1631 \times 10^{-5}$ & 0.132661 & 0.132918 & $2.5646 \times 10^{-4}$ \\
$\mathbf{0 . 2}$ & 0.082610 & 0.082760 & $1.4976 \times 10^{-4}$ & 0.248296 & 0.248746 & $4.4964 \times 10^{-4}$ \\
$\mathbf{0 . 3}$ & 0.109181 & 0.109438 & $2.5724 \times 10^{-4}$ & 0.354051 & 0.35463 & $5.7812 \times 10^{-4}$ \\
$\mathbf{0 . 4}$ & 0.125608 & 0.125981 & $3.7284 \times 10^{-4}$ & 0.455167 & 0.455819 & $6.5202 \times 10^{-4}$ \\
$\mathbf{0 . 5}$ & 0.131684 & 0.132166 & $4.8189 \times 10^{-4}$ & 0.554901 & 0.554593 & $6.9283 \times 10^{-4}$ \\
$\mathbf{0 . 6}$ & 0.127208 & 0.127772 & $5.644 \times 10^{-4}$ & 0.654458 & 0.655182 & $7.2408 \times 10^{-4}$ \\
$\mathbf{0 . 7}$ & 0.111981 & 0.112574 & $5.9319 \times 10^{-4}$ & 0.752936 & 0.753689 & $7.5278 \times 10^{-4}$ \\
$\mathbf{0 . 8}$ & 0.085810 & 0.086343 & $5.3337 \times 10^{-4}$ & 0.847262 & 0.848004 & $7.4246 \times 10^{-4}$ \\
$\mathbf{0 . 9}$ & 0.048528 & 0.048871 & $3.4651 \times 10^{-4}$ & 0.932713 & 0.932713 & $5.7590 \times 10^{-4}$ \\
$\mathbf{1 . 0}$ & 0.000000 & $5.98 \times 10^{-17}$ & $5.98 \times 10^{-17}$ & 1 & 1 & 0 \\
\hline & & & & & & \\
\hline
\end{tabular}

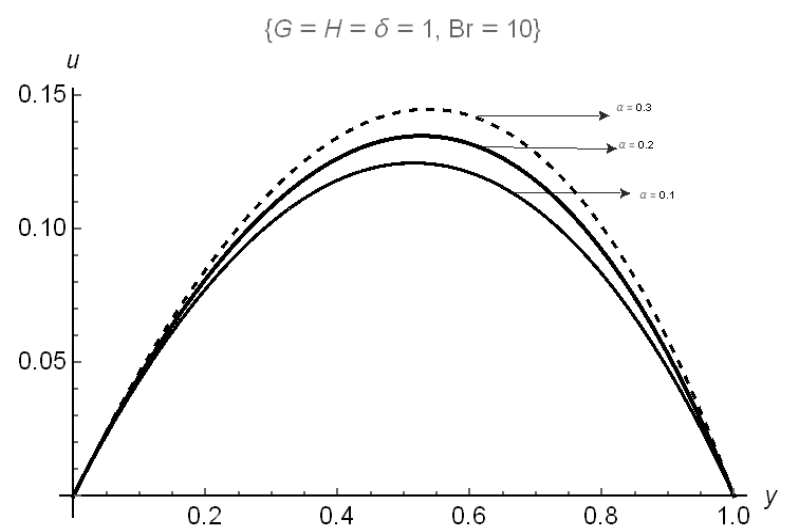

Figure 2: Velocity profile with change in $\alpha$.

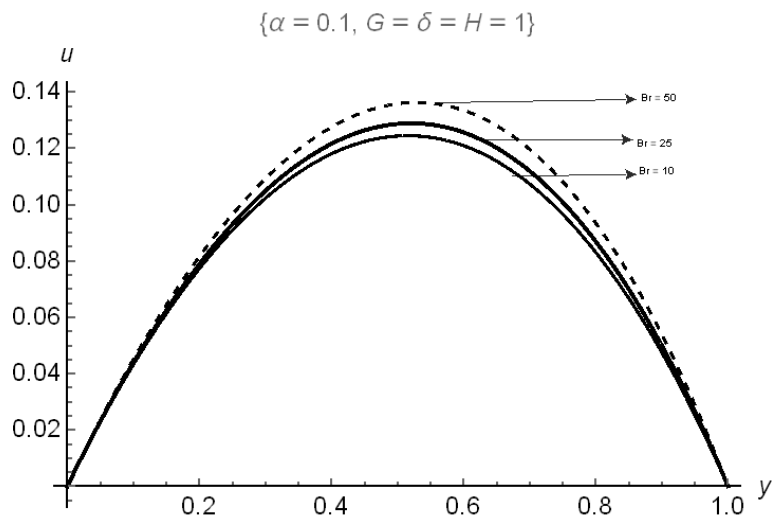

Figure 4: Velocity profile with change in $B r$.

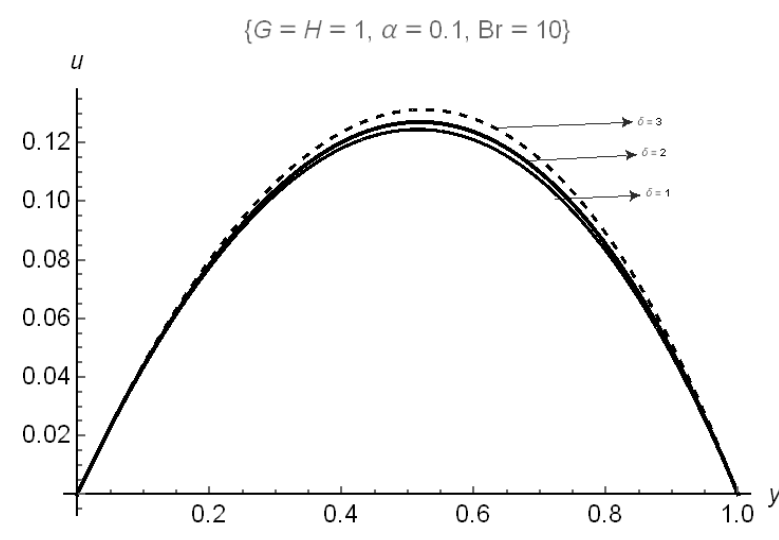

Figure 3: Velocity profile with change in $\delta$.

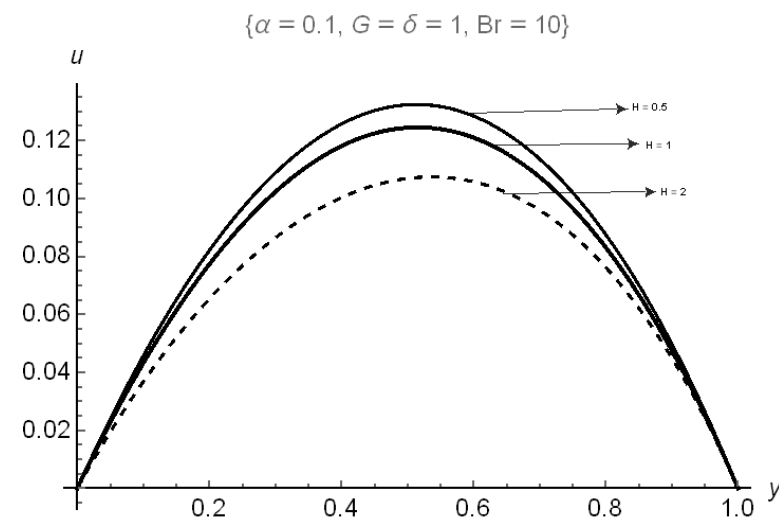

Figure 5: Velocity profile with change in $H$.

The temperature profiles of the fluid flow are displayed in figures 6 to 9. The observation showed that the maximum temperature varied at the centreline and maintains equilibrium at both the upper and lower wall channels with different temperatures stated in the boundary conditions (15) and (16). The increasing values of $\delta, H$ and $\mathrm{Br}$ in figures 6 to 8 make the temperature to rise due to interaction of the fluid in the channel while the viscosity variation parameter $(\alpha)$ tries to reduce the temperature at the centre as the value increases as shown in figure 9. 


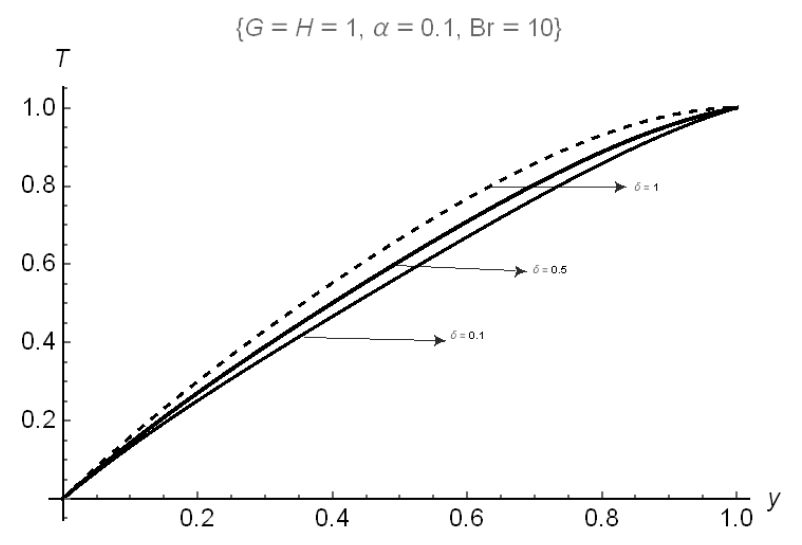

Figure 6: Temperature profile with change in $\delta$.

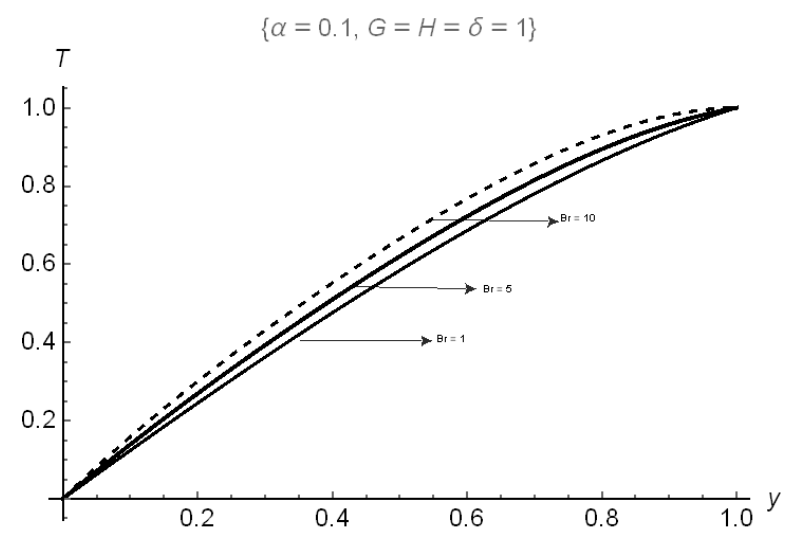

Figure 8: Temperature profile with change in $B r$.

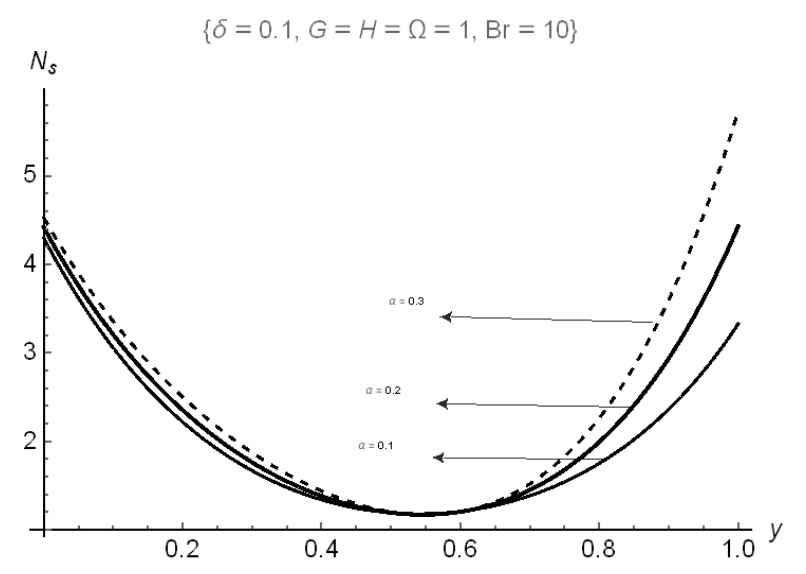

Figure 10: Entropy generation rate with change in $\alpha$.

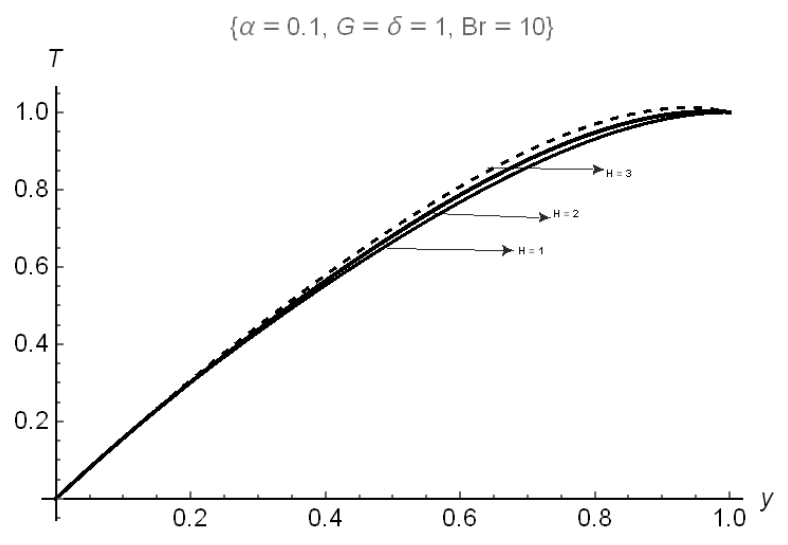

Figure 7: Temperature profile with change in $H$.

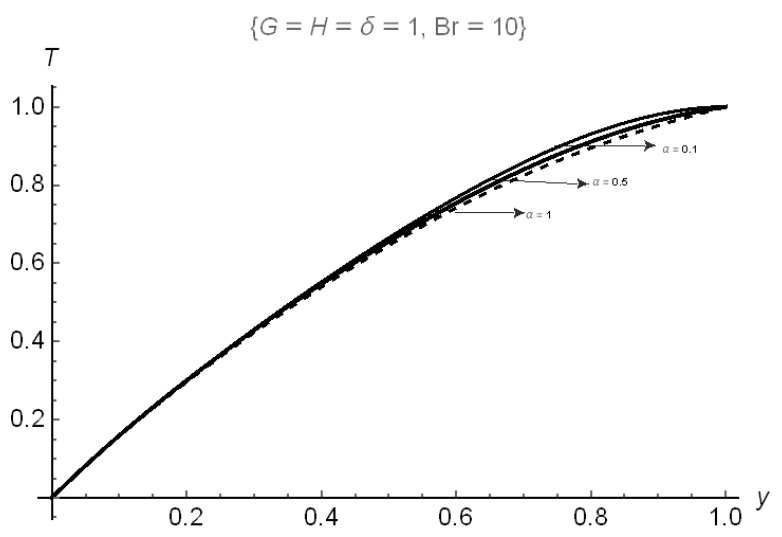

Figure 9: Temperature profile with change in $\alpha$.

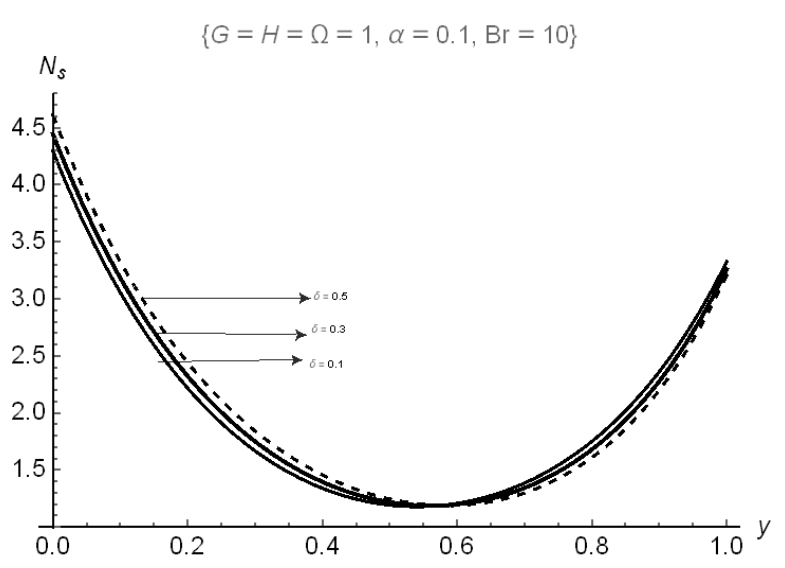

Figure 11: Entropy generation rate with change in $\delta$.

The rates of entropy production in the fluid flow are illustrated in figures 11 to 13 . The rate is maximum at both lower and upper plate surfaces and increases with greatest values of $\alpha$ and $\delta$ in figures 10 and 11 while the reverse is discovered in figure 12 with the wall temperature parameter $(\Omega)$. Meanwhile, in figure 13, the rate of entropy distribution reduces at both the lower and upper plate surfaces with rising values of $H$ but increases at around the central region with rising values of $H$. This showed that more disturbances occurred at the core region due to the influence of magnetic strength. 


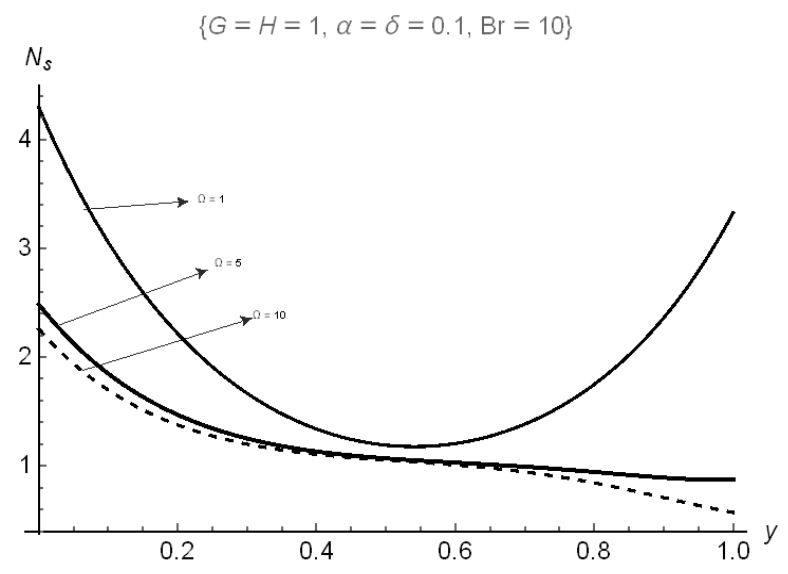

Figure 12: Entropy generation rate with change in $\Omega$.

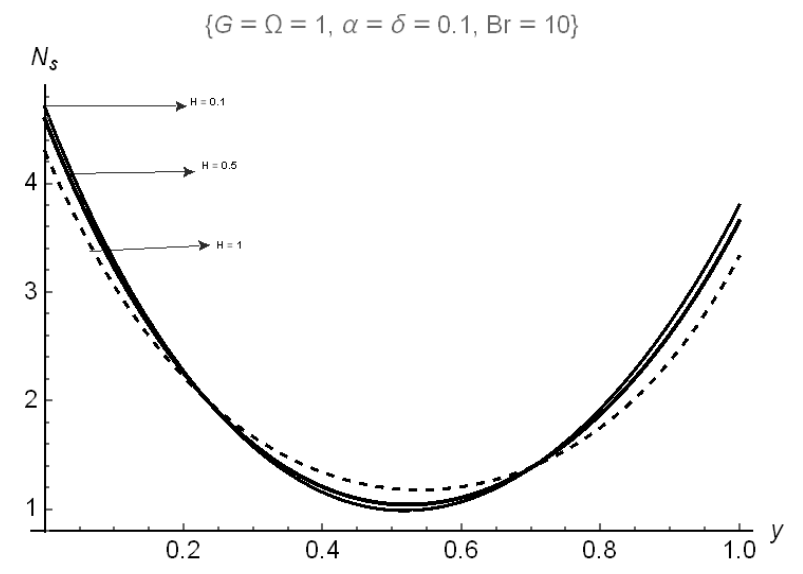

Figure 13: Entropy generation rate with change in $H$.

Figures 14 to 17 represent the graphical presentation of Bejan number of the fluid flow. The heat transfer irreversibility dominates at the central region where maximum value is recorded and goes down the plates surfaces. The increasing values of $\alpha$ reduce the dominion in figure 14. In figure 15, the dominion increases with rising values of $H$ at the lower wall, reduces at the central region and maintain equilibrium at the upper plate surface. Moreover, in figure 16, the heat transfer irreversibility dominates at the central region where maximum value is noticed with rising values of $(\Omega)$ across the flow channel. Meanwhile, the heat transfer irreversibility dominates at the lower wall with increasing values of internal heat generation $(\delta)$, maintain equilibrium at the central region and reduces at the upper plate surface.

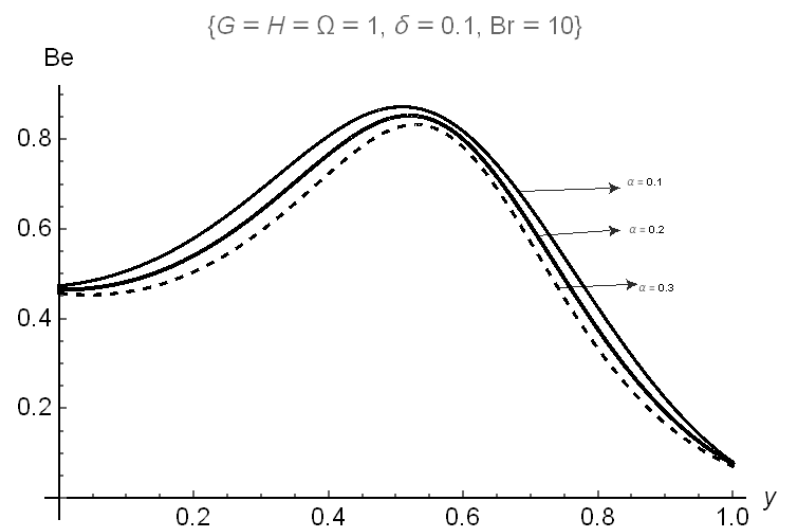

Figure 14: Bejan number with change in $\alpha$.

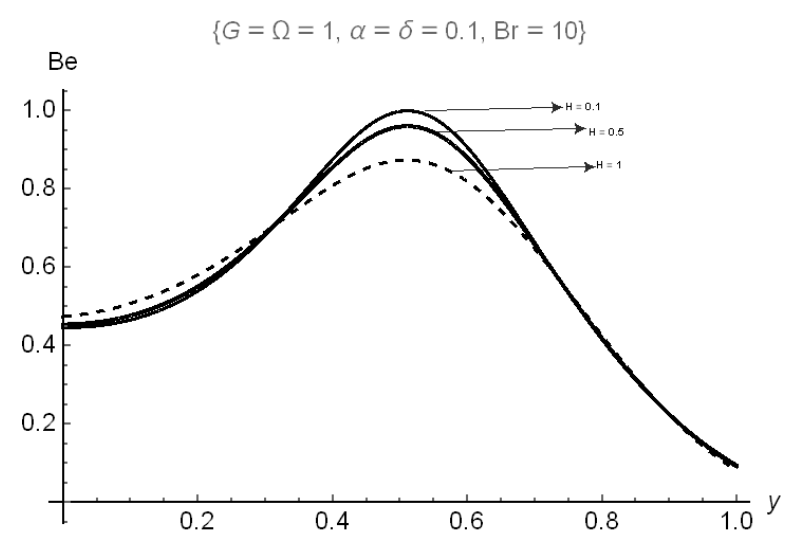

Figure 15: Bejan number with change in $H$.

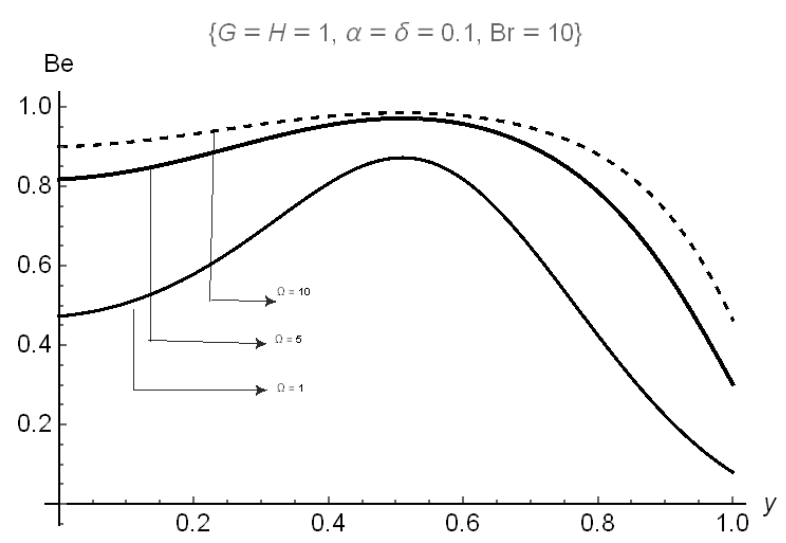

Figure 16: Bejan number with change in $\Omega$.

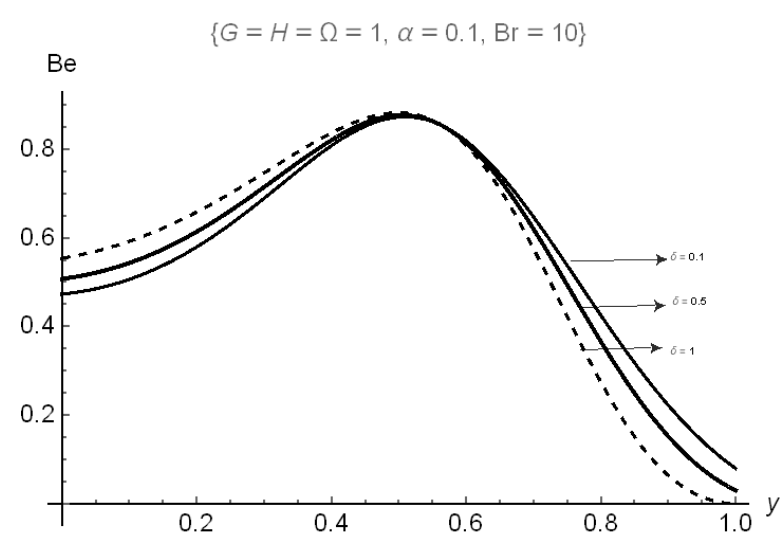

Figure 17: Bejan number with change in $\delta$. 


\section{CONCLUSION}

In this study, a critical examination on the impact of magnetic strength and internal heat generation on a variable-viscosity fluid flow with non-uniform wall temperature that varies linearly is carried out. The analytical expressions for the fluid velocity and temperature distributions are secured using Adomian decomposition method (ADM) and the results showed that the increasing parameters of viscous - variation, internal heat generation and Brinkman allow the fluid to interact and hence increase the fluid internal energy by moving faster while the magnetic strength parameter has a retarding effect due to the presence of Lorentz forces across the flow channel. Also, the increasing values of heat source, magnetic strength and Brinkman number make the temperature to rise due to interaction of the fluid in the channel while the viscosity - variation parameter reduces the fluid temperature. On a general note, the results significantly emphasized the effects of magnetic strength and heat source which cannot be neglected as it plays a major role in engineering and industrial applications.

\section{NOMENCLATURE}

$\begin{array}{llll}a & \text { Channel width } & L & \text { Channel characteristic length } \\ B_{0} & \text { Magnetic field strength } & T_{u} & \text { Upper wall temperature } \\ T_{L} & \text { Lower wall temperature } & \mu & \text { Temperature dependent parameter } \\ \rho & \text { The fluid density } & G & \text { Pressure gradient } \\ \Omega & \text { Wall temperature difference parameter } & \mathcal{E} & \text { Channel aspect ratio } \\ K & \text { Thermal conductivity } & p & \text { The pressure } \\ \sigma & \text { Electrical conductivity } & u & \text { The axial velocity } \\ c_{p} & \text { The specific heat at constant pressure } & v & \text { The normal velocity } \\ U & \text { Velocity scale } & P e & \text { Peclet number } \\ \alpha & \text { Viscosity - variation parameter } & H & \text { Hartmann number } \\ \delta & \text { Internal heat generation parameter } & \mathrm{Br} & \text { Brinkman number } \\ R e & \text { Reynolds number } & & \end{array}$

\section{References:}

[1] BABOlian, E., BiAZAR, J. (2002): Solution of nonlinear equations by modified Adomian decomposition method. Applied Mathematics and Computation 132 (1): 167-172. doi: 10.1016/S0096-3003(01)00184-9

[2] BAOKU, I.G., IsRael-CoOKey, C., Olajuwon, B.I. (2012): Magnetic field and thermal radiation effects on steady hydromagnetic Couette flow through a porous channel. Surveys in Mathematics and its Applications 1 (5): 215-228. doi:

[3] BeJan, A. (1996): Entropy-generation minimization. New York CRC Press, New York.

[4] EL-Amin, M.F. (2004): Combined effect of internal heat generation and magnetic field on free convection and mass transfer flow in a micro polar fluid with constant suction. Journal of Magnetism and Magnetic Materials 270 (1-2): 130-135. doi: 10.1016/j.jmmm.2003.08.011 
[5] ElbASHBESHY, E.M.A., BAZID, M.A.A. (2004): Heat transfer in a porous medium over a stretching surface with internal heat generation and suction or injection. Applied mathematics and computation 158 (3): 799-807. doi: 10.1016/j.amc.2003.08.141

[6] Hassan, A.R., Gbadeyan, J.A. (2015a): Entropy generation analysis of a reactive hydromagnetic fluid flow through a channel. U.P.B. Sci. Bull. Series A 77 (2): 285-296.

[7] Hassan, A.R., GBADEYAN, J.A., (2015b): A reactive hydromagnetic internal heat generating fluid flow through a channel. International Journal of Heat and Technology 33 (3): 43-50. doi: 10.18280/ijht.330306

[8] Hassan, A.R., MARITZ, R. (2016a): The analysis of a reactive hydromagnetic internal heat generating Poiseuille fluid flow through a channel. Springer plus 5: 1332. doi: 10.1186/s40064-016-2964-0

[9] HASSAN, A.R., MARITZ, R. (2016b): The analysis of a reactive hydromagnetic fluid flow in a channel through porous medium with convective cooling. U.P.B. Sci. Bull. Series D 78 (4): 43-56.

[10] Hassan, A.R., MARITZ, R. (2017a): The analysis of a variable-viscosity fluid flow between parallel porous plates with non-uniform wall temperature. Italian Journal of Pure and Applied Mathematics 38: 419-431.

[11] HaSsan, A.R., MARITZ, R. (2017b): The effect of internal heat generation on a steady hydromagnetic Poiseuille fluid flow between two parallel porous plates. Kragujevac Journal of Science 39: 37-46. doi: 10.5937/KgJSci1739037H

[12] JHA, B.K., AJIBADE, A.O. (2009): Free convective flow of heat generating/absorbing fluid between vertical porous plates with periodic heat input. International Communications in Heat and Mass Transfer 36 (6): 624-631.

doi: 10.1016/j.icheatmasstransfer.2009.03.003

[13] KIM, C.N. (2014): Magnetohydrodynamic flows entering the region of a flow channel insert in a duct. Fusion Engineering and design 89 (1): 56-68.

doi: 10.1016/j.fusengdes.2013.11.013

[14] Krishnamurthy, M.R., Prasannakumara, B.C., Gireesha, B.J., Gorla, R.S.R. (2015): Effect of viscous dissipation on hydromagnetic fluid flow and heat transfer of nanofluid over an exponentially stretching sheet with fluid-particle suspension. Cogent Mathematics 2: 1-18. doi: 10.1080/23311835.2015.1050973

[15] MAKINDE, O.D. (2008): Entropy-generation analysis for variable-viscosity channel flow with non-uniform wall temperature. Applied Energy 85 (5): 384-393.

doi: 10.1016/j.apenergy.2007.07.008

[16] Makinde, O.D., EegunjobI, A.S., TsheHLA, M.S. (2015): Thermodynamics analysis of a variable viscosity hydromagnetic Couette flow in a rotating system with hall effects. Entropy 17 (11): 7811-7826. doi: 10.3390/e17117811

[17] Olanrewaju, P.O., Gbadeyan, J.A., Hayat, T., Hendi, A.A. (2011): Effects of internal heat generation, thermal radiation and buoyancy force on a boundary layer over a vertical plate with a convective surface boundary condition. South African Journal of Science 107 (9/10): 80-85.

[18] PRASAD, B.G., KUMAR, A. (2011): Flow of a hydromagnetic fluid through porous media between permeable beds under exponentially decaying pressure gradient. Computational Methods in Science and Technology, 17 (1-2): 63-74. 
[19] RAY, S.S. (2014): New approach for general convergence of the Adomian decomposition method. World Applied Sciences Journal 32 (11): 2264-2268.

doi: 10.5829/idosi.wasj.2014.32.11.1317

[20] SAHA, S., ChaKRABARTI, S. (2013): Impact of magnetic field strength on magnetic fluid flow through a channel. International Journal of Engineering Research and Technology (IJERT) 2 (7): 1-8.

[21] Saravanan, S., Kandaswamy, P. (2004): Hydromagnetic stability of convective flow of variable viscosity fluids generated by internal heat sources. Z. angew. Math. Phys. 55: 451-467. doi: 451-467. 0044-2275/04/030451-17

[22] Sheikholeslami, M., Ganji, D.D., Rashidi, M.M. (2016): Magnetic field effect on unsteady nanofluid flow and heat transfer using Buongiorno model. Journal of Magnetism and Magnetic Materials 416: 164-173. doi: 10.1016/j.jmmm.2016.05.026

[23] UwANTA, I.J., HAMZA M.M. (2016): Unsteady/steady hydromagnetic flow of reactive viscous fluid in a vertical channel with thermal diffusion and temperature dependent properties. Journal of Applied Fluid Mechanics 9 (1): 169-176. doi: 10.18869/acadpub.jafm.68.224.23991

[24] WazWAZ, A.M. (1999): A reliable modification of Adomian decomposition method. Applied Mathematics and Computation 102 (1): 77-86. doi: 10.1016/S00963003(98)10024-3

[25] WAZWAZ, A.M. (2000): A new algorithm for calculating Adomian polynomials for nonlinear operators. Abstract and Applied Analysis 111 (1): 53-69. doi: 10.1016/S00963003(99)00063-6

[26] Wazwaz, A.M., El-Sayed, S.M. (2001): A new modification of the Adomian decomposition method for linear and nonlinear operators. Applied Mathematics and Computation 122 (3): 393-405. doi: 10.1016/S0096-3003(00)00060-6

[27] Woods, L.C. (1975): The thermodynamics of fluid systems. Oxford University Press, Oxford.

[28] XIAO, X., KIM, C.N. (2014): Magnetohydrodynamic flows in a hairpin duct under a magnetic field applied perpendicular to the plane of flow. Applied Mathematics and Computation 240: 1-15. doi: 10.1016/j.amc.2014.04.049

[29] Zelalem, Y., Mekonnen, S., Taddesse, A., Ahmed, M. (2013): Hydro magnetic effects on the flow of couple stress fluid through an extended porous channel. Journal of Global Research in Mathematics Archives 1 (10): 1-14. 